\title{
Crecimiento postraumático y variables psicosociales en víctimas de violencia política
}

\section{Posttraumatic growth and psychosocial variables among victims of political violence}

\author{
Mauricio C. Gómez ${ }^{\mathrm{a}}$, Felipe E. García ${ }^{\mathrm{a}, *}$ \\ ${ }^{a}$ Universidad de Concepción, Chile
}

\section{Resumen}

Antecedentes: la dictadura militar chilena (1973-1990) dejó miles de víctimas entre afectados directos y familiares, quienes pudieron desarrollar diversas secuelas en su salud mental. Objetivos: el presente estudio buscó evaluar un modelo predictivo de crecimiento postraumático (CPT) que incluía la ideología política, el afrontamiento religioso y la rumiación, en víctimas de la violencia política durante la dictadura. Método: participaron 200 personas adultas reconocidas en el Programa de Reparación y Atención Integral de Salud (PRAIS), encargada de brindar apoyo a las víctimas directas de violaciones a los derechos humanos durante la dictadura, y a sus familiares. De ellos 104 son mujeres (52\%) y 96 hombres (48\%), en un rango de edad entre los 30 y 91 años. Se utilizaron como instrumentos el Inventario de Crecimiento Postraumático, la Escala Breve de Afrontamiento Religioso, la Escala de Rumiación relacionada con el Evento y la Escala de Ideología Política. Resultados: los principales resultados indicaron que el afrontamiento religioso positivo y la rumiación deliberada predijeron el CPT. La rumiación deliberada media parcialmente entre el afrontamiento religioso positivo y el CPT. Conclusión: se concluye que el afrontamiento religioso positivo y el procesamiento cognitivo deliberado favorecen los cambios positivos en víctimas directas de la violencia política y sus familiares. Ante esto, se refuerza la idea que las víctimas de estas violaciones logran crecer después de estas experiencias dolorosas.

Palabras clave: afrontamiento religioso; crecimiento postraumático; ideología política; rumiación; víctimas de violencia política.

Para citar este artículo:

Gómez, M. C., \& García, F. E. (2021). Crecimiento postraumático y variables psicosociales en víctimas de violencia política. Liberabit, 27(1), e447. https://doi.org/10.24265/liberabit.2021.v27n1.05

\begin{abstract}
Background: The Chilean military dictatorship (1973-1990) left thousands of victims among those directly affected and their families, who were able to develop various mental health sequelae. Objective: The present study sought to evaluate a predictive model of posttraumatic growth (PTG) that included political ideology, religious coping and rumination in victims of political violence during the dictatorship. Method: The study involved 200 adults recognised in the Programme for Reparation and Integral Health Care (PRAIS), which is responsible for providing support to direct victims of human rights violations and their family members during the dictatorship. Out of these, 104 were women (52\%) and 96 were men (48\%) ranging in age from 30 to 91 years. The Posttraumatic Growth Inventory, the Brief Religious Coping Scale, the Event-Related Rumination Scale and the Political Ideology Scale were used as instruments. Results: The main results indicated that positive religious coping and deliberate rumination predicted PTG. Deliberate rumination partially mediates between positive religious coping and PTG. Conclusion: It is concluded that positive religious coping and deliberate cognitive processing favour positive change in those directly affected by political violence and their family members. This reinforces the idea that victims of these violations manage to grow after these painful experiences.
\end{abstract}

Keywords: Religious coping; posttraumatic growth; political ideology; rumination; victims of political violence.

Este es un artículo Open Access publicado bajo la licencia Creative Commons Atribución 4.0 Internacional. (CC-BY 4.0)

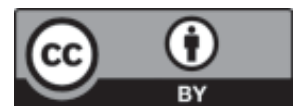

Universidad de San Martín de Porres, Lima - Perú

* fgarciam@udec.cl 


\section{Introducción}

El periodo más difícil para la construcción democrática de América Latina fue la década de los 70, caracterizada por regímenes militares, primero en Paraguay y Brasil, luego otros países como Perú, Bolivia, Uruguay, Chile y Argentina. En este periodo histórico estas dictaduras mantuvieron un control social basado en la Doctrina de Seguridad Nacional, utilizada por las Fuerzas Armadas (Faúndez \& Cornejo, 2010). En Chile, los efectos del terrorismo ejercido por la dictadura cívico-militar son aún visibles a más de 46 años después del golpe de Estado que derrocó al presidente Salvador Allende (Cárdenas et al., 2017). La estadística oficial reportó 28459 víctimas de privación de libertad y tortura (Comisión Nacional sobre Prisión Política y Tortura, 2004, 2005).

La dictadura chilena aplicó sistemáticamente torturas, homicidios, secuestros, desapariciones, exilios, relegaciones, hostigamientos y allanamientos masivos. De las víctimas reconocidas en la Corporación Nacional de Reparación y Reconciliación (1996), el $50.2 \%$ militaba en partidos de izquierda, mientras que el 46\% no tenía militancia política (Valdivia, 2003).

Diversos estudios en Chile coinciden en señalar que la magnitud de las alteraciones psicológicas depende en gran medida de la vulneración recibida, la biografía personal, el desarrollo social del individuo, el desenvolvimiento político del afectado y el significado de esta experiencia (Becker et al., 1990; Gómez, 1985; Lira, 1990); generando reacciones que afectaban la salud mental, como depresión, trastornos del sueño, reacciones angustiosas, entre otras (Faúndez \& Cornejo, 2010).

En la década del 90, tras el fin de la dictadura, el Ministerio de Salud implementó el Programa de Reparación y Atención Integral de Salud (PRAIS) el cual reconoce el daño transgeneracional extendiendo sus prestaciones de salud a las tres generaciones de las víctimas directas (Hails, 2009). En los estudios de morbilidad PRAIS se destaca que el $43 \%$ de las familias habrían sufrido una experiencia traumática, el $30 \%$ fueron afectadas por dos experiencias y un $19 \%$ por tres o más experiencias traumáticas (Minoletti, 2002).

A pesar del daño producido por la violación de los derechos humanos (DD. HH.), se ha sugerido que el impacto negativo de la violencia no es irreversible (Arnoso et al., 2011). Las personas que enfrentan grandes crisis de la vida suelen experimentar emociones angustiantes, ansiedades y miedos específicos como reacciones típicas; pero también pueden experimentar transformaciones positivas, entre ellas la posibilidad de crecer a partir de la experiencia traumática vivida, lo que se ha denominado crecimiento postraumático (CPT).

El CPT hace referencia al cambio positivo que un individuo experimenta como resultado del proceso de lucha que emprende a partir de la vivencia de un suceso traumático (Calhoun \& Tedeschi, 1999). El CPT se divide en tres grandes categorías que pueden experimentar las personas: a) cambios en uno mismo, por ejemplo, percibirse como más seguro de sí mismo para enfrentar situaciones difíciles; b) cambios en las relaciones interpersonales, por ejemplo, dar más valor que antes a las redes de apoyo; y c) cambios en la espiritualidad y en la filosofía de vida, por ejemplo, dar un mayor valor a la propia existencia (Calhoun \& Tedeschi, 2001).

El CPT es un constructo poco estudiado en situaciones de terrorismo de Estado (Cárdenas et al., 2016). Algunos ejemplos de los estudios realizados son los siguientes. En Colombia, 316 jóvenes víctimas de violencia política experimentaron cambios positivos en las relaciones interpersonales con familiares y grupo de pares (Morales et al., 2019). En una muestra ecuatoriana, 48 víctimas de tortura y privación de libertad también lograron cambios a nivel espiritual y en la filosofía de vida (Reyes et al., 2018). En Perú, los familiares de detenidos y desaparecidos experimentaron mayor capacidad para establecer relaciones significativas dentro de la misma agrupación, favoreciendo el CPT (Zambrano, 2020). 
En Chile, víctimas del terrorismo de Estado desarrollaron CPT mediante la rumiación deliberada (Cárdenas et al., 2016). Los estudios mencionados sugieren que los métodos ejercidos por la violencia política no han logrado destruir las convicciones políticas de las personas, pues de alguna manera han logrado CPT (Cárdenas et al., 2017).

La ideología política (IP) es un constructo que alude a creencias sobre una visión del mundo, la cual moldea el comportamiento político de las personas (Brussino et al., 2011). La importancia de examinar la IP y la posición política es sugerida por la teoría de gestión del terror, la cual sostiene que la ideología se vuelve más prominente cuando hay mayor conciencia de la muerte, disminuyendo los elementos estresantes del entorno amenazante, proporcionando significado y orden en el caos (Oren \& Possick, 2010).

En un estudio basado en la teoría de la gestión del terror, con 2999 jóvenes israelitas, mostró que el compromiso ideológico contribuyó a mejorar el nivel de bienestar ante la adversidad y lograr CPT (Laufer et al., 2010). Incluso, la ubicación ideológica tiende a polarizarse o hacerse más intensa en situaciones de amenaza, como se ha observado en población norteamericana (Gadarian, 2010), suiza (Chatard et al., 2010) e israelí (Laor et al., 2010).

El modelo de IP como cognición social motivada logra distinguir dos dimensiones centrales en la tensión entre el progresismo o liberalismo (izquierda) y el conservadurismo (derecha): a) abogar frente a resistir el cambio social y b) rechazar frente a aceptar la desigualdad (Jost et al., 2003). Las personas que sostienen ideas progresistas denotan apertura a nuevas experiencias, asociadas a una mayor complejidad cognitiva, flexibilidad, tolerancia a la incertidumbre y mayor autoestima (Jost \& Amodio, 2012). Los conservadores se destacan por una mayor estabilidad ideológica (Schildkraut et al., 2021) y sus valores normativos de estabilidad personal, logro y poder (Brussino et al., 2013); sostienen la creencia de que las personas tienen más libertades que otros y que estas relaciones tienen un orden jerárquico (Delfino \& Zubieta, 2011).

Los estudios empíricos en la IP y su relación con el trauma son escasos, a diferencia del estudio de otro sistema de creencias, como el religioso, donde existe mayor conocimiento sobre su rol ante el trauma (Oren \& Possick, 2009). Tanto la IP como la religiosidad son cosmovisiones culturales que proporcionan valores, como una forma de vida, y comportamiento ético que pueden tener un efecto positivo en tiempos de adversidad (Chong et al., 2017).

Para Laufer et al. (2010), las creencias religiosas tienen tanta relevancia como la IP a manera de mediadoras del efecto del miedo sobre el CPT en contextos de violencia social. La teoría de la gestión del terror de Greenberg et al. (2003) puede ayudar a entender la influencia de las creencias religiosas al igual como lo hace con la IP. Un ejemplo es el estudio del afrontamiento religioso y el trauma. Pargament et al. (1998) distinguen entre el afrontamiento religioso positivo y el afrontamiento religioso negativo. El primero se relaciona a la satisfacción psicológica espiritual, a la búsqueda de apoyo espiritual, a la búsqueda de ayuda de clérigos o miembros de la iglesia y al perdón religioso. En cambio, el segundo se refiere a una valoración religiosa punitiva, a la insatisfacción espiritual y a la insatisfacción religiosa interpersonal. De acuerdo a los estudios asociados a esta teoría, el papel del afrontamiento religioso positivo facilita la reducción de la angustia por la muerte. En cambio, el afrontamiento religioso negativo suele estar acompañado de estados de angustia y ansiedad ante factores estresantes que condicionen una mayor vulnerabilidad (Mohammadzadeh \& Najafi, 2020). Los estudios han sido consistentes en mostrar que el afrontamiento religioso negativo puede predecir la sintomatología postraumática y un afrontamiento religioso positivo puede predecir el CPT (García et al., 2020; Gerber et al., 2011).

Otro elemento importante en la comprensión de los procesos psicológicos que conducen al CPT es 
el trabajo cognitivo que puede ayudar a restaurar o revisar el mundo de supuestos que ha sido desafiado por el evento estresante (Cann et al., 2010). El modelo teórico de Calhoun et al. (2010) propone que, para lograr un crecimiento tras un evento traumático, los procesos más involuntarios de pensamiento repetitivo deberían dar paso a procesos más voluntarios (García, Vega et al., 2018). Una rumiación más deliberada relacionada con el evento es probable que ya no se enfoque hacia aspectos negativos, lo que permite que eventualmente la persona desarrolle CPT; en cambio, la rumiación intrusiva relacionada con el evento es más probable que esté relacionada con sintomatología postraumática (García, Vega et al, 2018).

Por lo tanto, la relevancia de la IP es que la gente tiende a aferrarse a sus creencias políticas, abrazando a los individuos que son similares a ellos (el grupo interno) y rechazando a los que son diferentes (el grupo externo). Al aumentar la conciencia de la muerte, por ejemplo, en el caso de ser vulnerados en sus DD. HH. por parte del Estado, el papel de la IP se vuelve más prominente, ya que sirve como amortiguador de los factores estresantes (Laufer \& Solomon, 2010). Del mismo modo, el afrontamiento religioso positivo está más fuertemente relacionado con el CPT y el afrontamiento religioso negativo con la sintomatología postraumática (García et al., 2020; Gerber et al., 2011; Prati \& Pietrantoni, 2009). En ese proceso, la rumiación cognitiva podría conducir a la comprensión de un suceso traumático, desde la restauración de creencias fundamentales anteriores o a la reconstrucción de creencias fundamentales revisadas, con una visión más optimista a través del CPT (Cárdenas et al., 2016). Concretamente, la rumiación deliberada se relaciona más con el CPT en experiencias altamente estresantes (García, Barraza et al., 2018; Stockton et al., 2011; Su \& Chen, 2015), mediando la relación entre las estrategias de afrontamiento utilizadas y el CPT, como se ha observado en varios estudios (e.g., García et al., 2016). De ese modo, el CPT pone en marcha la creación de un sentido de vida como respuesta al trauma político (Cárdenas et al., 2016).
El objetivo principal de este estudio es evaluar un modelo que incluye la ideología política, el afrontamiento religioso y la rumiación para la predicción del CPT en víctimas de terrorismo de Estado. Asimismo, se evaluará el rol mediador de la rumiación deliberada en la relación entre el afrontamiento religioso positivo y el CPT. Se espera que el modelo evaluado sea significativo y que la rumiación deliberada medie parcial o totalmente la relación entre afrontamiento religioso positivo y CPT.

\section{Método}

\section{Diseño}

El diseño del estudio fue de tipo cuantitativo, descriptivo, correlacional y transversal.

\section{Participantes}

Participaron 200 personas adultas pertenecientes al registro del PRAIS. El programa PRAIS es un programa del Estado orientado a atender problemas de salud en víctimas de la dictadura militar y sus familiares directos. Los criterios de inclusión fueron hombres y mujeres que hayan nacido antes o durante la dictadura militar, que terminó en el año 1989, y ser usuario activo del PRAIS del Servicio de Salud Arauco, en el sur de Chile. Los participantes debían ser víctimas directas, afectados directos o beneficiarios, de acuerdo a la clasificación de PRAIS (Minoletti, 2002): a) las víctimas directas son aquellos afectados por tortura, prisión política, exilio, relegación, clandestinidad por persecución política y exoneración por causa política; b) los afectados directos incluyen a quienes vivían y formaban su grupo familiar inmediato, por ejemplo, la madre, padre, hijo o hija, hermano o hermana, cónyuge o conviviente; c) beneficiarios son las personas que no vivieron el evento represivo de manera directa, pero son familiares nacidos o poseen alguna afiliación con los «afectados directos», incorporándose nietos o nietas, sobrinos o sobrinas, nueras o yernos. 
Se excluyeron 6 usuarios que presentaron problemas cognitivos y 40 personas por falta de conectividad a internet, lo que impidió que participaran del estudio.

Del total de la población, 96 fueron hombres (48\%) y 104 mujeres (52\%), en un rango de edad entre $30 \mathrm{y}$ 91 años $(M=55.56 ; D E=11.58)$. Con respecto al estado civil, el 59.5\% de los participantes eran casados, $17.5 \%$ separados y divorciados, $16.5 \%$ solteros y $6.5 \%$ viudos. En cuanto al nivel ocupacional, 21.5\% son trabajadores independientes, $15.5 \%$ dueña de casa, $15 \%$ pensionados por la Ley Valech (que estableció beneficios a víctimas de tortura y/o prisión política durante la dictadura), $14.5 \%$ trabajadores dependientes y otros oficios el 33.5\%.

El muestreo fue no probabilístico, por conveniencia de accesibilidad.

\section{Instrumentos}

Cuestionario socio-demográfico. Constó de preguntas relacionadas con la edad, género, estado civil, ocupación, ubicación ideológica, pertenencia religiosa y relación con el evento traumático, por ejemplo, víctima directa, cónyuge, hijo, etc. Esta última pregunta está respaldada en la norma técnica nacional PRAIS, $N .^{\circ} 88$, que califica como víctimas hasta tres generaciones.

Inventario de Crecimiento Postraumático, versión breve (PGTI- SF). Creado por Cann et al. (2010) y validada para su uso en población chilena por García y Wlodarczyk (2016). Está compuesto de 10 ítems que se responden en una escala Likert de seis alternativas, desde 0 (ningún cambio) hasta 5 (un cambio muy importante). En el estudio de García y Wlodarczyk (2016) se obtuvo una consistencia interna del instrumento $\alpha=.94$. Asimismo, mostró evidencia de validez de constructo a través del análisis factorial confirmatorio (AFC). En el presente estudio, el instrumento obtuvo una consistencia interna de $\alpha=.84$.

\section{Escala de Rumiación Relacionada al Evento} (ERRI). Esta escala fue creada por Cann, et al. (2011) $\mathrm{y}$ mide pensamientos repetitivos, intrusivos y deliberados. Fue adaptada para su uso en población chilena por Alzugaray et al. (2015), quienes presentaron una versión más breve de 12 ítems, siete para la subescala de rumiación intrusiva (RI) y cinco para la subescala de rumiación deliberada (RD). Se responde en una escala Likert desde 0 (casi nunca) a 3 (casi siempre). La subescala RI mostró una $\alpha=$ .91 y la subescala de RD un $\alpha=.85$. Además, se realizó un AFE para validez de constructo, encontrándose los dos factores claramente diferenciados y correlacionados positivamente entre sí $(r=.60)$. La subescala RI mostró para este estudio un $\alpha=.81$ y la subescala de RD, un $\alpha=.91$.

Escala Brief-RCOPE. Fue elaborado por Pargament et al. (1998), utilizado para la medición de patrones positivos y negativos del afrontamiento religioso. Fue validado para su uso en población chilena por García et al. (2020). Tiene dos subescalas de siete ítems cada una: afrontamiento religioso positivo (ARP) $\mathrm{y}$ afrontamiento religioso negativo (ARN). Se responde en una escala Likert desde 0 (nada) a 3 (mucho). La subescala ARP obtuvo un $\alpha=.94$; mientras que el ARN, un $\alpha=$.79. Este instrumento posee propiedades psicométricas aceptables de validez de convergencia y predictiva para población chilena. En el presente estudio, la subescala ARP obtuvo un $\alpha=.91 \mathrm{y}$ la subescala ARN un $\alpha=.70$.

Escala de Ideología Política (EIP). Fue construido por Brussino et al. (2011) para estudiar la ideología política sobre temas de actualidad en América Latina. Para este estudio se utilizaron dos dimensiones generales: conservadurismo y progresismo. El insumo consta de 22 ítems situacionales, los cuales permiten a la persona puntuar desde 1 (nada de acuerdo) hasta 5 (totalmente de acuerdo). En el estudio original las consistencias internas fluctuaron entre $\alpha=.66$ y $\alpha=.77$. En el actual estudio se obtuvo una consistencia interna para el conservadurismo $\alpha=.80$ y el progresismo $\alpha=.73$. 


\section{Procedimiento}

Se realizó una aplicación piloto a 10 participantes con el fin de verificar la comprensibilidad del set de instrumentos y determinar el tiempo de aplicación considerando un formato virtual. A partir de los resultados, se incorporó una nueva pregunta de religión durante el periodo de dictadura en el cuestionario sociodemográfico, se modificó el ítem N. ${ }^{\circ} 10$ en la escala de ideología política (que aludía a la opinión sobre la regulación del Estado sobre la producción de soja y se cambió por producción forestal, puesto que en la región de Arauco no se produce soja) y se estimó un tiempo de aplicación entre 20 y 25 minutos. Se concluyó, además, que era viable la administración de las encuestas por internet a través de formularios Google.

Los instrumentos fueron aplicados entre julio y septiembre de 2020, los participantes fueron contactados tras la revisión de las fichas clínicas del programa PRAIS (morbilidad, odontológico, salud mental y procedimientos de alto costo), para luego consultar la participación vía telefónica. Posteriormente, las encuestas fueron enviadas a cada correo electrónico del usuario/a para ser contestado a través de formularios Google. Todas las alternativas tenían carácter de obligatoriedad, evitando omisión de respuestas y quedando respaldado vía correo electrónico del investigador principal.

El formulario contenía el consentimiento informado, objetivos y características del estudio. La participación en el estudio no representaba un riesgo físico o psicológico, pero podía influir en una posible alteración emocional ante recuerdos dolorosos. Para ello, no solo se informó sobre esta eventualidad en el consentimiento, sino que además se realizó seguimiento los primeros tres meses después de la recolección de datos para evitar una posible reexperimentación postraumática, además de evaluar la salud mental en general debido a la pandemia por coronavirus.

El presente estudio contó con la aprobación de la Comisión de Ética Científico del Servicio de Salud
Arauco de Chile y el Departamento de Gestión de Calidad y Seguridad del Paciente con fecha del 19 de febrero de 2020 y N. resolución 290.

\section{Análisis de datos}

Primero, se realizó un análisis descriptivo de las variables independientes y dependientes. Luego, para probar las hipótesis del estudio, se utilizó el coeficiente de correlación de Pearson y la regresión lineal múltiple.

Por último, se llevó a cabo un análisis bootstrap para comprobar los efectos indirectos del afrontamiento religioso positivo sobre el crecimiento postraumático mediado por la rumiación deliberada, según las directrices propuestas por Mathieu y Taylor (2006). Para ello, se utilizó el modelo 4 del módulo Process, utilizando la evaluación de una mediación simple. Se realizó una estimación bootstrap de sesgo corregido con un intervalo de confianza del 95\% usando 5000 muestras bootstrap. En este caso, la mediación existe si el intervalo de confianza no es cero.

Para la realización del análisis de datos, se utilizó el software estadístico IBM SPSS Statistics 25. La mediación se evaluó utilizando el módulo Process para SPSS.

\section{Resultados}

La Tabla 1 muestra los estadísticos descriptivos para evaluar puntajes mínimos, máximos, media y desviación estándar.

Se evaluaron las diferencias en los niveles del CPT entre las distintas categorías incluidas en el cuestionario socio-demográfico, para lo cual se usaron $t$ de Student o ANOVA usando la prueba post hoc HSD de Tukey, a modo de controlar la influencia de alguna variable relevante en los siguientes análisis.

Como resultado, no se encontraron diferencias significativas en el género, estado civil, ocupación, 
personas con familiares enfermos en cuarentena, estrés, familiares contagiados producto del COVID19, y ubicación política durante y después la dictadura.
En la Tabla 2, se observa las correlaciones bivariadas entre las variables del estudio.

Tabla 1

Estadísticos descriptivos y de las variables del estudio

\begin{tabular}{lcccc}
\hline \multicolumn{1}{c}{ Variables } & Mínimo & Máximo & Media & $D E$ \\
\hline Crecimiento postraumático & 0 & 50 & 31.30 & 7.37 \\
Conservadurismo & 12 & 56 & 31.68 & 9.94 \\
Progresismo & 17 & 50 & 34.47 & 8.27 \\
Rumiación deliberada & 0 & 14 & 6.73 & 3.02 \\
Rumiación intrusiva & 0 & 18 & 4.28 & 3.34 \\
AR positivo & 0 & 20 & 8.89 & 5.49 \\
AR negativo & 0 & 12 & 1.96 & 2.33
\end{tabular}

Nota: AR = Afrontamiento religioso.

Fuente: Elaboración propia.

Tabla 2

Correlaciones de Pearson entre las variables de estudio

\begin{tabular}{lcccccc}
\hline \multicolumn{1}{c}{ Variables } & 2 & 3 & 4 & 5 & 6 & 7 \\
\hline 1. Progresismo & $-.65^{* * *}$ & $-.35^{* * *}$ & $-.31^{* * *}$ & $.18^{* *}$ & $.29^{* * *}$ & .03 \\
2. Conservadurismo & - & $.36^{* * *}$ & $.38^{* * *}$ & -.03 & $-.18^{*}$ & .08 \\
3. AR positivo & & - & $.47^{* * *}$ & $.24^{* * *}$ & .01 & $.48^{* * *}$ \\
4. AR negativo & & - & $.16^{*}$ & .10 & $.14^{*}$ \\
5. Rumiación deliberada & & & & - & $.62^{* * *}$ & $.36^{* * *}$ \\
6. Rumiación intrusiva & & & & - & $.21^{* *}$ \\
7. Crecimiento postraumático & & & & & - & -
\end{tabular}

Nota: $* p<.05^{* *} ; p<.01^{* * *} ; p<.001$

Fuente: Elaboración propia.

En general, se observa que el CPT presenta su relación más fuerte con el afrontamiento religioso positivo $(r=-.48 ; p<.001)$ y luego con la rumiación deliberada $(r=-.36 ; p<.001)$, la rumiación intrusiva $(r=-.21 ; p<.01)$ y el afrontamiento religioso negativo $(r=-.14 ; p<.05)$. No se observa una relación significativa de progresismo y conservadurismo con el CPT.
Para evaluar la relación y el efecto de la ideología política progresista, ideología política conservadora, afrontamientos religioso positivo, afrontamiento religioso negativo, rumiación deliberada e intrusiva sobre el CPT, se llevó a cabo un análisis de regresión lineal múltiple (RLM), como se observa en la Tabla 3. 
Tabla 3

Coeficientes, valor t y significancia del modelo RLM de las variables independientes sobre el CPT

\begin{tabular}{lrccc}
\hline & \multicolumn{2}{c}{ Coeficientes no estandarizados } & \multicolumn{2}{c}{ Coeficientes tipificados } \\
\cline { 2 - 5 } & $\beta$ & Error típ. & $\beta$ & Valor $t$ \\
\hline (Constante) & 16.20 & 3.96 & & $4.086^{* * *}$ \\
Conservadurismo & .04 & .06 & .05 & .615 \\
Progresismo & .14 & .07 & .15 & 1.854 \\
Rumiación deliberada & .45 & .20 & .18 & $2.302^{*}$ \\
Rumiación intrusiva & .15 & .17 & .07 & .856 \\
AR positivo & .70 & .10 & .52 & $7.171^{* * *}$ \\
AR negativo & -.35 & .22 & -.11 & -1.580 \\
\hline
\end{tabular}

Nota: $* p<.05 ; * * * p<.001$

Fuente: Elaboración propia.

El modelo evaluado presentó un coeficiente $R^{2}=$ .32 , es decir, explica el $32 \%$ de la varianza. Se observa que la rumiación deliberada $(\beta=.18)$ y el afrontamiento religioso positivo $(\beta=.52)$ son las únicas variables que influyen significativamente sobre el CPT.

Para evaluar el rol mediador de la rumiación deliberada entre el afrontamiento religioso positivo y el CPT, se realizó la estimación bootstrap. El efecto indirecto estandarizado se estimó que estaba en el intervalo de confianza entre .02 y .14 $(M=.06 ; E E$ $=.03)$. Dado que el cero no está en el $95 \%$ del intervalo de confianza, se puede concluir que el efecto indirecto fue significativo con un valor $p<.001$ y que la relación entre el afrontamiento religioso positivo y el CPT está parcialmente mediado por la rumiación deliberada (véase la Figura 1).

\section{Figura 1}

Modelo de mediación

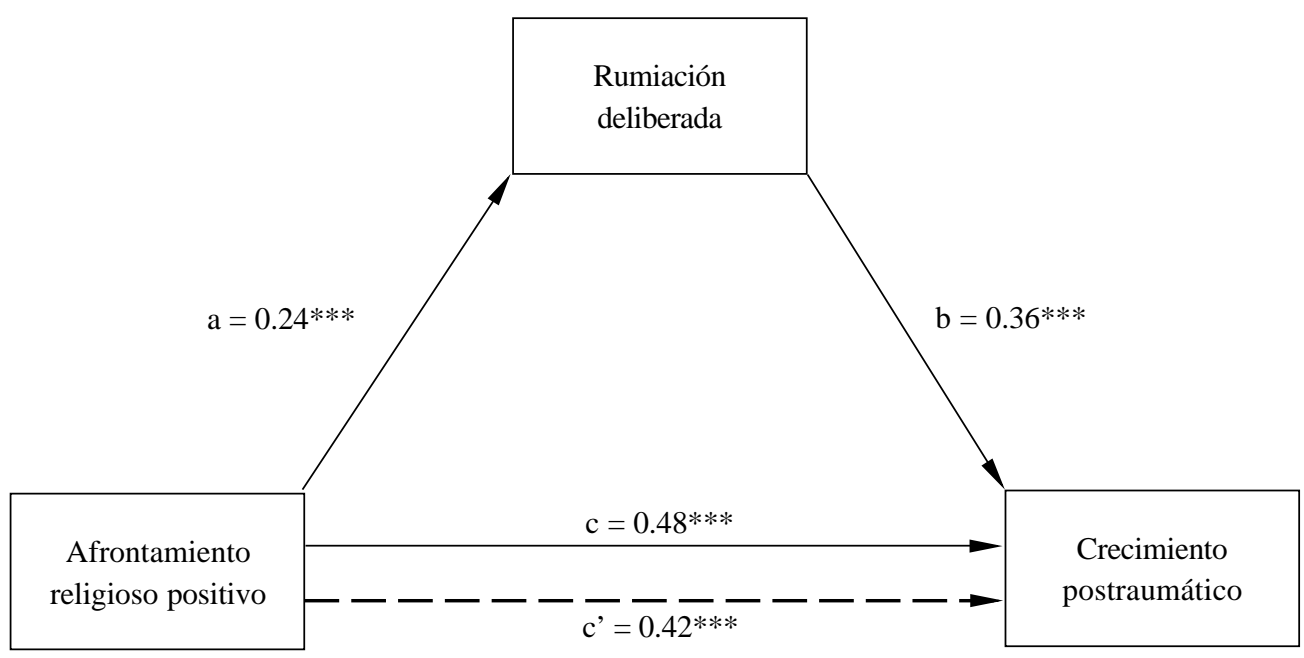

Nota: Se muestran los coeficientes de regresión estandarizados; *** $p<.01$

Fuente: Elaboración propia. 


\section{Discusión}

El objetivo de este estudio era determinar la relación entre el CPT, la ideología política, el afrontamiento religioso y la rumiación en víctimas de violencia política de la dictadura militar chilena que gobernó de facto entre los años 1973 y 1990. Se encontró que ni el progresismo ni el conservadurismo se relaciona con el CPT, pero el afrontamiento religioso positivo y la rumiación deliberada sí se relacionan.

La relación positiva observada del afrontamiento religioso positivo y el CPT es concordante con la literatura científica (Gerber et al., 2011; Prati \& Pietrantoni, 2009; Shaw et al., 2005). Estudios realizados en Chile con personas afectadas por desastres naturales establecen que el afrontamiento religioso positivo se relaciona en forma directa con el CPT (Avilés et al., 2014; García, Páez et al., 2014) e incluso la predice longitudinalmente (García et al., 2020). Esta fuerte relación puede deberse a que las construcciones religiosas tienden a ser vitales para definir el significado y la importancia de los eventos de la vida para muchas personas, especialmente, frente a eventos que alteran negativamente la vida (Pargament, 1997). Se puede concluir que el afrontamiento religioso positivo sirve como una estrategia que permite una adaptación psicológica saludable a través del tiempo mejorando los niveles del CPT (García et al., 2020).

La relación directa observada de la rumiación deliberada y el CPT se ha encontrado en muchos estudios (Cárdenas et al., 2016; García et al., 2016; Stockton et al., 2011). La rumiación deliberada se asocia a una forma más productiva de procesamiento cognitivo en la persona después de un trauma. Aparentemente, este procesamiento cognitivo es necesario para identificar cambios positivos y percibir crecimiento ante hechos negativos, actuando como mediador entre la severidad subjetiva del evento o las estrategias de afrontamiento utilizadas con el CPT (García, Jaramillo et al., 2014; García et al., 2016; García \& Miranda, 2019).
Ante esto, se hipotetizó que la rumiación deliberada también mediaría la relación entre el afrontamiento religioso positivo y el CPT, encontrándose en este estudio una mediación parcial, pues al incorporar el mediador, el afrontamiento religioso positivo sigue teniendo una influencia directa significativa con el CPT, pero disminuye esta influencia. Es de suponer que el afrontamiento religioso positivo influye en forma directa sobre el CPT a través de la asignación de significado que provee una creencia religiosa para comprender experiencias extremas (García et al., 2020), tal como la tortura o el encarcelamiento injustificado. También lo hace en forma indirecta a través de las reflexiones voluntarias que la persona ejerce para comprender lo vivido y afrontar sus consecuencias, algo que es posibilitado por la rumiación deliberada (Cárdenas et al., 2016; García et al., 2016).

En tanto, la ideología política progresista o conservadora no se relaciona con el CPT, contradiciendo una de las hipótesis de este estudio. Esto podría deberse a que los progresistas tienen menos atributos de coherencia ideológica y estabilidad en la medición de la IP (Brussino, 2016) y los conservadores son menos consistentes en sus preferencias políticas (Schildkraut et al., 2021). Además, gran parte de los chilenos dejaron la militancia política en 1973 y para los años 90 surge una despolitización de la sociedad como herencia de la dictadura chilena, la cual mermó la participación política de la ciudadanía (Valdivia, 2013; Vallejos, 2014), por lo que probablemente en la actualidad la ideología tiene escasa influencia como factor protector ante la adversidad.

Una de las limitaciones del estudio es el tiempo transcurrido desde el evento traumático, como fueron las violaciones a los DD. HH. ocurridas entre el año 1973 y el año 1990. Si bien se midió el crecimiento actual ante esta experiencia, muchas otras variables medidas, como el posicionamiento político de entonces o el afrontamiento religioso empleado, pueden estar afectadas por el sesgo del recuerdo. Haber utilizado un muestreo por conveniencia ha sido una segunda limitación, pues los participantes no necesariamente 
representan al universo de víctimas de la violencia política. Esta falta de representatividad se ve potenciada debido a que la recolección de datos se realizó en los primeros meses de la pandemia por Covid-19 en Chile vía internet. La tercera limitante es la naturaleza transversal del estudio, lo que impide concluir relaciones de causa y efecto en el tiempo, aunque las relaciones predichas se basaban en teoría y evidencia empírica previa. La cuarta limitante fue la naturaleza multifacética de la ideología política que requiere del uso de varios indicadores para ser capturado correctamente y no solo el elegido en el presente estudio.

Por tal motivo, para estudios futuros se sugiere incorporar otras medidas relacionadas a la ideología política, como el compromiso ideológico y la tendencia de voto. Así mismo, se sugiere incorporar evaluaciones sobre emociones, como afectividad positiva y negativa y regulación emocional, debido a la coexistencia de emociones positivas y negativas que se han observado en el individuo que presenta CPT (Calhoun \& Tedeschi, 1999).

En Latinoamérica, continente azotado por una serie de dictaduras que ejercieron represión política sobre sus habitantes, tras la recuperación de la democracia, se realizaron diversas acciones destinadas a reparar el daño causado. Para ello, se establecieron Comisiones de Verdad para abordar los impactos de la represión política en los distintos países, como Perú, Guatemala, El Salvador, Uruguay, Ecuador, entre otros, las mismas que tuvieron un rol fundamental en la reparación del daño y en la búsqueda de una reconciliación nacional. En este sentido, el programa PRAIS, que nace a partir del trabajo de una de estas comisiones, ha contribuido a la superación de las secuelas adversas producto de los hechos traumáticos (Barrera, 2017; Hails, 2009). Los resultados obtenidos en el presente estudio no solo serían un aporte al conocimiento sobre el CPT, sino también a la línea de estudios que abordan las secuelas de la violencia política en Latinoamérica. Además, podrían contribuir en la implementación de directrices terapéuticas, enfocándose en la persona como un agente promotor de cambios y dotado de derechos sociales, promoviendo a su vez la recuperación de la memoria histórica del país (Heida, 2019).

\section{Conflicto de intereses}

Los autores declaran no tener conflictos de interés de ningún tipo.

\section{Responsabilidad ética}

En este estudio no se realizaron experimentos de ningún tipo. El procedimiento fue realizado a través de la aplicación de instrumentos en formato de autoinforme vía virtual, sin manipulación de las variables.

\section{Contribución de la autoría}

MGR: concepción y diseño del estudio, recolección datos, interpretación de los datos, discusión y escritura del manuscrito.

FEG: concepción y diseño del estudio, análisis de los datos, interpretación de los datos, discusión y revisión final del manuscrito.

\section{Confidencialidad de los datos}

El estudio se realizó siguiendo los protocolos del Ministerio de Salud y el código de ética del Colegio de Psicólogos, ambos de Chile. Contó con la aprobación de la Comisión de Ética Científico del Servicio de Salud Arauco de Chile y el Departamento de Gestión de Calidad y Seguridad del Paciente con fecha 19/02/2020 y resolución N. 290.

\section{Derecho a la privacidad y consentimiento informado}

La participación fue de tipo voluntaria previa lectura y aprobación de una carta de consentimiento. La aplicación de las encuestas se realizó en forma anónima, las respuestas de cada participante fueron 
almacenadas con un código y la información ha sido resguardada por el primer autor de este estudio.

\section{Agradecimientos}

Agradecemos al Servicio de Salud Arauco y, en especial, al Programa PRAIS por permitirnos el acceso a los participantes. Manifestamos, además, nuestro reconocimiento a todas las víctimas directas e indirectas de la dictadura militar que gobernó Chile desde el año 1973 hasta el año 1990 y que hasta el día de hoy sufren las consecuencias de las graves vulneraciones a los DD. HH. en sí mismos y en sus seres queridos. Este estudio nos permite de alguna forma recuperar y conservar la memoria histórica en nuestra sociedad actual.

\section{Referencias}

Alzugaray, C., García, F., Reyes, A., \& Álvarez, R. (2015). Propiedades psicométricas de una versión breve de la escala de rumiación relacionada a un evento en población chilena afectada por eventos altamente estresantes. Ajayu, 13(2), 183-198.

Arnoso, M., Bilbao, M. A., Páez, D., Iraurgi, I., Kanyangara, P., Rimé, B., Pérez-Sales, P., MartínBeristain, C., Díaz, D., \& Blanco, A. (2011). Violencia colectiva y creencias básicas sobre el mundo, los otros y el yo: impacto y reconstrucción. En D. Páez, C. Martín-Beristain, J. L. González, N. Basabe, \& J. de Rivera (Eds.), Superando la violencia colectiva y construyendo cultura de paz (pp. 247-277). Editorial Fundamentos.

Avilés, P., Cova, F., Bustos, C., \& García, F. (2014). Afrontamiento y rumiación frente a eventos adversos y crecimiento postraumático en estudiantes universitarios. Liberabit, 20(2), 281-292.

Barrera, E. A. (2017). Las políticas de reparación en Chile: El Caso del Programa de Reparación y Ayuda integral en Salud y Derechos Humanos (PRAIS), Valdivia (2001-2016) [Tesis de Licenciatura, Universidad Austral de Chile]. http://cybertesis.uach.cl/ tesis/uach/2017/ffy.22p/doc/ffy.22p.pdf

Becker, D., Castillo M. I., Gómez, E., Kovalskys, J., \& Lira, E. (1990). Psicopatología y proceso psicoterapéutico de situaciones políticas traumáticas. En I. Martín-Baró (Ed.), Psicología Social de la Guerra: Trauma y Terapia (pp. 46-52). UCA Editores.

Brussino, S. (2016). El desarrollo histórico de la psicología política en Latino-América y Argentina. En S. Brussino (Coord.), Políticamente, Contribuciones desde la Psicología Política en Argentina (pp. 13-36). CONICET - Consejo Nacional de Investigaciones Científicas y Técnicas.

Brussino, S., Imhoff, D., Rabbia, H. H., \& Paz, A. P. (2013). Ideología política en torno a Issues y valores sociales: un estudio correlacional en ciudadanos de Córdoba/ Argentina. América Latina Hoy, 65, 161-182. http:// dx.doi.org/10.14201/alh201365161182

Brussino, S. A., Rabbia, H. H., Imhoff, D., \& García, A. P. P. (2011). Dimensión operativa de la ideología política en ciudadanos de Córdoba - Argentina. Psicología Política, 43, 85-106.

Cann, A., Calhoun, L. G., Tedeschi, R. G., \& Solomon, D. T. (2010). Posttraumatic Growth and Depreciation as Independent Predictors of Well-Being. Journal of Loss and Trauma, 15(3), 156-166. https://doi.org/10.1080/ 15325020903375826

Cann, A., Calhoun L., Tedeschi R., Triplett K., Vishnevsky T., \& Lindstrom, C. (2011). Assessing Posttraumatic Cognitive Processes: The Event Related Rumination Inventory, Anxiety, Stress, \& Coping, 24(2), 137-156. https://doi.org/10.1080/10615806.2010.529901

Calhoun, L. G., Cann, A., \& Tedeschi, R. G. (2010). The Posttraumatic Growth Model: Sociocultural Considerations. En T. Weiss, \& R. Berger (Eds.), Posttraumatic Growth and Culturally Competent Practice (pp. 1-14). John Wiley \& Sons Inc.

Calhoun, L. G., \& Tedeschi, R. G. (Eds.) (1999). Facilitating Posttraumatic Growth: A Clinician's Guide. Lawrence Erlbaum Associates Publishers. https://doi.org/10.4324/ 9781410602268

Calhoun, L. G., \& Tedeschi, R. G. (2001). Posttraumatic Growth: The Positive Lesson of Loss. En Neimeyer, R. A. (Ed.), Meaning Construction and the Experience of Loss.

Cárdenas, M., Arnoso, M., \& Faúndez, A. (2016). Deliberate Rumination and Positive Reappraisal as 
Serial Mediators between Life Impact and Posttraumatic Growth in Victims of State Terrorism in Chile (1973-1990). Journal of Interpersonal Violence, 34(3), 545-561. https://doi.org/10.1177/ 0886260516642294

Cárdenas, M., Faúndez, A., Arancibia, M., \& Ceruti, M. (2017). The Relationship Between Posttraumatic Growth and Psychosocial Variables in Survivors of State Terrorism and their Relatives. Journal of Interpersonal Violence, 36(1-2), 428-447. https://doi.org/10.1177/ 0886260517727494

Chatard, A., Arndt, J., \& Pyszczynski, T. (2010). Loss Shapes Political Views? Terror Management, Political Ideology, and the Death of Close Others. Basic and Applied Social Psychology, 32(1), 2-7. https://doi.org/ 10.1080/01973530903539713

Chong, G., Mohamed, S., Hatim, A., \& Zuraida, N. (2017). Anxiety and Depression in Cancer Patients: The Association with Religiosity and Religious Coping. Journal of Religion and Health, 56(2), 575-590. https:/ /doi.org/10.1007/s10943-016-0267-y

Comisión Nacional sobre Prisión Política y Tortura. (2004). Informe de la Comisión Nacional sobre Prisión Política y Tortura. Gobierno de Chile.

Comisión Nacional sobre Prisión Política y Tortura. (2005). Informe complementario de la Comisión Nacional sobre Prisión Política y Tortura. Gobierno de Chile.

Corporación Nacional de Reparación y Reconciliación. (1996). Informe sobre calificación de las víctimas de violaciones de derechos humanos y de la violencia política. Gobierno de Chile. http://www.archivochile. com/Derechos_humanos/Com_Rettig/hhddrettig 0017.pdf

Delfino, G. I., \& Zubieta, E. M. (2011). Valores y política. Análisis del perfil axiológico de los estudiantes universitarios de la ciudad de Buenos Aires (República Argentina). Interdisciplinaria, 28(1), 93-114.

Faúndez, X., \& Cornejo, M. (2010). Aproximaciones al estudio de la Transmisión Transgeneracional del Trauma Psicosocial. Revista de Psicología, 19(2), 3154. http://dx.doi.org/10.5354/0719-0581.2010.17107

Gadarian, S. K. (2010). The Politics of Threat: How Terrorism News Shapes Foreign Policy Attitudes. The
Journal of Politics, 72(2), 469-483. https://oi.org/ $10.1017 / \mathrm{s} 0022381609990910$

García, F. E., Barraza, C. G., Wlodarczyk, A., \& Reyes, A. R. (2018). Psychometric Properties of the Brief-COPE for the Evaluation of Coping Strategies in the Chilean Population. Psicologia: Reflexão e Crítica, 31(22). https://doi.org/10.1186/s41155-018-0102-3

García, F. E., Cova, F., Rincón, P., Vázquez, C., \& Páez, D. (2016). Coping, Rumination and Posttraumatic Growth in People Affected by an Earthquake. Psicothema, 28(1), 59-65. https://doi.org/10.7334/psicothema 2015.100

García, F. E., Jaramillo, C., Martínez, A., Valenzuela, I., \& Cova, F. (2014). Respuestas psicológicas ante un desastre natural: estrés y crecimiento postraumático. Liberabit, 20(1), 121-130.

García, F. E., \& Miranda, F. (2019). Predictores de sintomatología postraumática aguda y crecimiento postestrés tras un accidente laboral reciente. Actualidades en Psicología, 33(126), 117-135. https://doi.org/ 10.15517/ap.v33i126.32680

García, F. E., Oyanedel, J. C., Páez, D., \& Arias, P. R. (2020). Psychometric Properties of the Brief Religious Coping Scale (Brief-RCOPE) in Chilean Adults Exposed to Stressful Events. Journal of Religion and Health, 60, 475-487. https://doi.org/10.1007/s10943-019-00976-7

García, F. E., Páez-Rovira, D., Cartes, G., Neira, H., \& Reyes, A. (2014). Religious Coping, Social Support and Subjective Severity as Predictors of Posttraumatic Growth in People Affected by the Earthquake in Chile on 27/2/2010. Religions, 5(4), 1132-1145. https://doi.org/ 10.3390/rel5041132

García, F. E., Vega, N., Briones, F., \& Bulnes, Y. (2018). Rumiación, crecimiento y sintomatología postraumática en personas que han vivido experiencias altamente estresantes. Avances en Psicología Latinoamericana, 36(3), 443-457. https://doi.org/10.12804/revistas.uro sario.edu.co/apl/a.4983

García, F. E., \& Wlodarczyk, A. (2016). Psychometric Properties of the Posttraumatic Growth inventory-short Form among Chilean Adults. Journal of Loss and Trauma, 21(4), 303-314. https://doi.org/10.1080/ 15325024.2015.1108788 
Gerber, M. M., Boals, A., \& Schuettler, D. (2011). The Unique Contributions of Positive and Negative Religious Coping to Posttraumatic Growth and PTSD. Psychology of Religion and Spirituality, 3(4), 298-307. https://doi.org/10.1037/a0023016

Greenberg, J., Martens, A., Jonas, E., Eisenstadt, D., Pyszczynski, T., \& Solomon, S. (2003). Psychological Defense in Anticipation of Anxiety: Eliminating the Potential for Anxiety Eliminates the Effects of Mortality Salience on Worldview Defense. Psychological Science, 14(5), 516-519. https://doi.org/10.1111/14679280.03454

Gómez, E. (1985). La tortura como experiencia traumática: Perspectivas del daño [ponencia]. Seminario La Tortura en América Latina, Montevideo, Uruguay. http:/ /www.smu.org.uy/dpmc/pracmed/temas/ddhh/tortura/ a59-63.pdf

Hails, K. (2009). Las secuelas psicológicas y las necesidades en salud mental para las víctimas de la dictadura: una mirada crítica a los servicios de PRAIS. Cultura, Desarrollo y Justicia Social.

Heida, A. (2019). Dictadura militar y memoria histórica en Chile [tesis de maestría, Universidad de Leiden]. Repositorio de estudiantes. https://hdl.handle.net/ 1887/81303

Jost, J. T., \& Amodio, D. M. (2012). Political Ideology as Motivated Social Cognition: Behavioral and Neuroscientific Evidence. Motivation and Emotion, 36(1), 55-64. https://doi.org/10.1007/s11031-011-9260-7

Jost, J. T., Glaser, J., Kruglanski, A. W., \& Sulloway, F. J. (2003). Exceptions that Prove the Rule - Using a Theory of Motivated Social Cognition to Account for Ideological Incongruities and Political Anomalies: Reply to Greenberg and Jonas (2003). Psychological Bulletin, 129(3), 383-393. https://doi.org/10.1037/00332909.129.3.383

Laor, N., Yanay-Shani, A., Wolmer, L., \& Khoury, O. (2010). A Trauma-Like Model of Political Extremism: PsychoPolitical Fault Lines in Israel. Annals of the New York Academy of Sciences, 1208(1), 24-31. https://doi.org/ 10.1111/j.1749-6632.2010.05693.x

Laufer, A., \& Solomon, Z. (2010). Political Ideology and Psychological Symptoms Following Terror. Youth \&
Society, 41(3), 414-433. https://doi.org/10.1177/ 0044118X09333658

Laufer, A., Solomon, Z., \& Levine, S. Z. (2010). Elaboration on Posttraumatic Growth in youth Exposed to Terror: The Role of Religiosity and Political Ideology. Social Psychiatry and Psychiatric Epidemiology, 45(6), 647653. https://doi.org/10.1007/s00127-009-0106-5

Lira, E. (1990). Psicología del miedo y conducta colectiva en Chile. En I. Martin-Baro (Ed.), Psicología Social de la guerra: trauma y terapia (pp.22-25). UCA Editores.

Mathieu, J. E., \& Taylor, S. R. (2006). Clarifying Conditions and Decision Points for Mediational Type Inferences in Organizational Behavior. Journal of Organizational Behavior, 27(8), 1031-1056. https://doi.org/10.1002/ job.406

Minoletti, A. (2002). Norma técnica para la atención de personas afectadas por la represión política ejercida por el Estado en el período 1973-1990. Ministerio de Salud, Gobierno de Chile.

Mohammadzadeh, A., \& Najafi, M. (2020). The Comparison of Death Anxiety, Obsession, and Depression Between Muslim Population with Positive and Negative Religious Coping. Journal of Religion and Health, 59(2), 10551064. https://doi.org/10.1007/s10943-018-0679-y

Morales, L. C. B., Otero, M. M., \& Correa, M. N. (2019). Salud mental y crecimiento postraumático en jóvenes víctimas de violencia política en una institución educativa distrital de la Ciudad de Cartagena [tesis de grado, Universidad de San Buenaventura].

Oren, L., \& Possick, C. (2009). Religiosity and Posttraumatic Stress Following Forced Relocation. Journal of Loss and Trauma, 14(2), 144-160. https://doi.org/10.1080/ 15325020902724586

Oren, L., \& Possick, C. (2010). Is Ideology a Risk Factor for PTSD Symptom Severity among Israeli Political Evacuees? Journal of Traumatic Stress, 23(4), 483-490. https://doi.org/10.1002/jts.20540

Pargament, K. I. (1997). The Psychology of Religion and Coping. Theory, Research, Practice. Guilford Press.

Pargament, K. I., Smith, B. W., Koenig, H. G., \& Perez, L. (1998). Patterns of Positive and Negative Religious Coping with Major Life Stressors. Journal for the 
Scientific Study of Religion, 37(4), 710-724. https:// doi.org/10.2307/1388152

Prati, G., \& Pietrantoni, L. (2009). Optimism, Social Support, and Coping Strategies as Factors Contributing to Posttraumatic Growth: A Meta-Analysis. Journal of Loss \& Trauma, 14(5), 364-388. https://doi.org/10.1080/ 15325020902724271

Reyes, C., Grondona-Opazo, G., Rodríguez, M., \& Páez, D. (2018). Posttraumatic Growth of Victims Informed by the Truth Commission of Ecuador. Revista Interamericana de Psicología/Interamerican Journal of Psychology, 52(3), 379-388. https://doi.org/10.30849/ rip\%20ijp.v52i3.476

Schildkraut, D. J., Berry, J. M., \& Glaser, J. M. (2021). Ideological Bubbles and Two Types of Conservatives. Public Opinion Quarterly, 84(2), 508-522. https://doi. org/10.1093/poq/nfaa027

Shaw, A., Joseph, S., \& Linley, P. A. (2005). Religion, Spirituality, and Posttraumatic Growth: A Systematic Review. Mental Health, Religion \& Culture, 8(1), 111. https://doi.org/10.1080/1367467032000157981

Stockton, H., Hunt, N., \& Joseph, S. (2011). Cognitive Processing, Rumination, and Posttraumatic Growth. Journal of Traumatic Stress, 24(1), 85-92. https:// doi.org/10.1002/jts.20606
Su, Y. J., \& Chen, S. H. (2015). Emerging Posttraumatic Growth: A Prospective Study with pre-and Posttrauma Psychological Predictors. Psychological Trauma: Theory, Research, Practice, and Policy, 7(2), 103-111. https://doi.org/10.1037/tra0000008

Valdivia, V. (2003). Terrorism and Political Violence During the Pinochet Years: Chile, 1973-1989. Radical History Review, 85(1), 182-190. https://doi.org/10.1215/016365 45-2003-85-182

Valdivia, V. (2013). El Santiago de Ravinet: Despolitización y consolidación del proyecto dictatorial en el Chile de los noventa. Historia (Santiago), 46(1), 177-219. http://dx.doi.org/10.4067/S0717-71942013000100006

Vallejos, R. A. (2014). La nueva política en el Chile postdictatorial: ¿pasividad ciudadana o clientelismo desde abajo? (1990-1996). Estudos IberoAmericanos, 40(1), 169-189.

Zambrano, H. R. G. (2020). La importancia de lo relacional para el empoderamiento en justicia y verdad en miembros de una organización de familiares de desaparecidos por la violencia política en el Perú. Journal de Ciencias Sociales, 8(15), 147-172. https://doi.org/10.18682/jcs.vi15.998

\footnotetext{
Mauricio C. Gómez

Departamento de Psicología, Facultad de Ciencias Sociales, Universidad de Concepción.

Magíster en Psicología de la Salud, Universidad de Concepción.

ORCID: http://orcid.org/0000-0002-7965-7157

maugomez@udec.cl
}

Felipe E. García

Departamento de Psiquiatría y Salud Mental, Facultad de Medicina, Universidad de Concepción. Programa de Neurociencia, Psiquiatría y Salud Mental NEPSAM, Universidad de Concepción.

Doctor en Psicología, académico de la Universidad de Concepción. Su línea de investigación es el afrontamiento a situaciones de crisis, estrés y trauma, y las consecuencias de estos eventos en la salud mental, tanto negativa como positiva.

ORCID: http://orcid.org/0000-0002-4161-5840

Autor corresponsal: fgarciam@udec.cl 